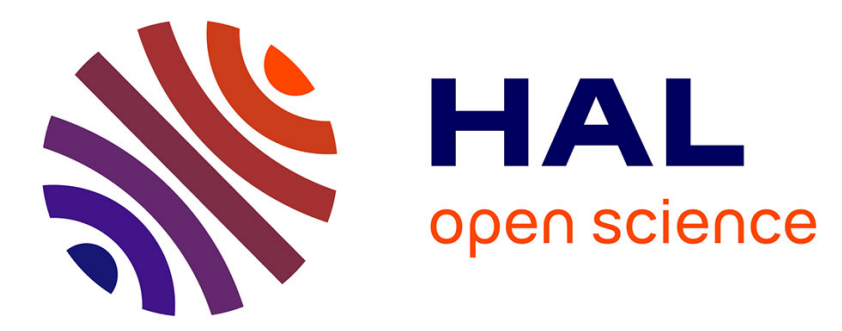

\title{
Direct experimental evidence for a negative heat capacity in the liquid-to-gas like phase transition in hydrogen cluster ions: backbending of the caloric curve
}

F. Gobet, B. Farizon, M. Farizon, M.J. Gaillard, J.P. Buchet, M. Carre, P. Scheier, T.D. Maerk

\section{To cite this version:}

F. Gobet, B. Farizon, M. Farizon, M.J. Gaillard, J.P. Buchet, et al.. Direct experimental evidence for a negative heat capacity in the liquid-to-gas like phase transition in hydrogen cluster ions: backbending of the caloric curve. Physical Review Letters, 2002, 89, pp.183403-1-183403-4. 10.1103/PhysRevLett.89.183403 . in2p3-00011856

\section{HAL Id: in2p3-00011856 \\ https://hal.in2p3.fr/in2p3-00011856}

Submitted on 22 Oct 2002

HAL is a multi-disciplinary open access archive for the deposit and dissemination of scientific research documents, whether they are published or not. The documents may come from teaching and research institutions in France or abroad, or from public or private research centers.
L'archive ouverte pluridisciplinaire HAL, est destinée au dépôt et à la diffusion de documents scientifiques de niveau recherche, publiés ou non, émanant des établissements d'enseignement et de recherche français ou étrangers, des laboratoires publics ou privés. 


\title{
Direct experimental evidence for a negative heat capacity in the liquid-to-gas like phase transition in hydrogen cluster ions: backbending of the caloric curve
}

\author{
F.Gobet, B.Farizon, M.Farizon, M.J.Gaillard \\ Institut de Physique Nucléaire de Lyon, IN2P3-CNRS et Université Claude \\ Bernard, 43 boulevard du 11 Novembre 1918, F-69622 Villeurbanne Cedex, France \\ J.P.Buchet, M.Carré, \\ Laboratoire de Spectrométrie Ionique et Moléculaire, CNRS UMR 5579 et Université \\ Claude Bernard, 43 boulevard du 11 Novembre 1918, F-69622 Villeurbanne Cedex, France \\ P.Scheier and T.D.Märk \\ Institut für Ionenphysik, Leopold Franzens Universität, Technikerstr.25, A-6020 Innsbruck, Austria
}

(Dated: 2th May 2002)

\begin{abstract}
By (i) selecting specific decay reactions in high energy collisions (60 keV/amu) of hydrogen cluster ions with a helium target (utilizing event-by-event data of a recently developed multi-coincidence experiment) and by (ii) deriving corresponding temperatures for these microcanonical cluster ensembles (analyzing respective fragment distributions) we are able to construct caloric curves for $\mathrm{H}_{3}^{+}\left(\mathrm{H}_{2}\right)_{m}$ cluster ions $(6 \leq m \leq 14)$. All individual curves and the mean of these curves show a backbending in the plateau region thus constituting direct evidence for a negative microcanonical heat capacity in the liquid-to-gas like transition of these finite systems.
\end{abstract}

PACS numbers: 36.40.Ei; 36.40.Qv

One of the most fascinating phenomena in the thermodynamics of complex systems is the prediction of a possible backbending in caloric curves in microcanonical ensembles. As this is counterintuitive to every day experience and as this is in contradiction to a theorem that specific heats are positive $[1,2]$ it was for a time seen as a real paradox in thermodynamics. This was first discussed in detail by Thirring [1] some time ago and has since been an accepted prediction in astronomy, where adding energy to a star or star cluster would make it expand and cool down [1-4]. Moreover, recently such an effect (increase of total energy causing a reduction in temperature) has been predicted by applying statistical thermodynamical methods to describe phase transitions of small systems, i.e., for the melting of atomic clusters [5-8] and the decay of hot nuclei [9].

It appears that Pochodzalla et al. [8] were the first to report a measured relation between the temperature of hot decaying nuclei resulting from $\mathrm{Au}+\mathrm{Au}$ collisions at $600 \mathrm{MeV} / \mathrm{amu}$ and the excitation energy, the shape of this relation exhibiting the characteristic plateau expected for a phase transition. Though being the first experimental observation of a caloric curve for a liquidto-gas phase transition for nuclear systems [8], the determination of the temperature was later questioned [9], in particular because later experiments arrived at different shapes some not even showing a plateau-like region (see [10] and [11] and references therein). Nevertheless, D'Agostino et al. [11] were even able recently to infer the existence of a negative heat capacity in excited nuclear systems $(\mathrm{Au}+\mathrm{Au}$ collisions at $35 \mathrm{AMeV})$ from the eventby-event study of energy fluctuations thus providing also in this way direct evidence for a first order liquid-to-gas like phase transition.

On the other hand, Haberland and co-workers [12] re- ported the first experimental determination of a caloric curve for the solid-to-liquid like transition (melting) of a small cluster, i.e., a sodium cluster ion consisting of 139 atoms. A beam of cluster ions was generated with a canonical distribution of internal energy thus fixing the temperature. One cluster size was selected (thus switching to microcanonical system), irradiated by photons and the photofragmentation pattern (whose positions can be related to the energy) is measured as a function of cluster temperature. Similarly, Bachels et al. [13] reported recently a caloric curve for a free tin cluster distribution (without mass selection) impinging on a sensitive pyroelectric foil, the interpretation of their experiment was, however, later questioned [14]. Furthermore, Haberland and co-workers [15] were recently able by extending their method to map out the energy distribution of the fragments demonstrating by the bimodal shape observed a possible existence of a negative heat capacity for a melting 147 atom sodium cluster. Haberland and co-workers have in addition very recently constructed the caloric curve across the liquid-to-gas like transition [16] using known data of the atomic gas. Moreover, at the same time, we reported [17] a first experimental signature of a caloric curve for a molecular cluster ion of rather small size, i.e., $\mathrm{H}_{3}^{+}\left(\mathrm{H}_{2}\right)_{12}$ showing by the occurrence of a plateau like shape in the caloric curve the typical prerequisites of a first order phase transition in a finite system.

Despite rapid progress in this field (see also [18]), clear evidence for the negative heat capacity in small systems is still missing, even more so when considering remarks in a recent review on critical phenomena in nuclear fragmentation by Bonasera et al [19] stating that "the temperatures measured from ratios containing these isotopes could be meaningless" and recommending that "the disagreement between experimental results (are large and) 
must be solved". Nevertheless, Chomaz et al. recently discussed the properties of caloric curves for a microcanonical lattice gas model [20] and predicted under certain circumstances the occurrence of backbending in the microcanonical liquid-gas phase transition.

Therefore, the aim of the present study was to ascertain a first direct experimental fingerprint of the occurrence of backbending in the caloric curve measured for a finite system and thus providing a direct proof for the existence of a negative heat capacity in a phase transition. In order to monitor here directly the negative heat capacity it was necessary based on our first attempt to derive a caloric curve for decaying $\mathrm{H}_{3}^{+}\left(\mathrm{H}_{2}\right)_{12}$ ions in [17] to (i) improve our data analysis in terms of accuracy (analysing more data in a more accurate way for each collision) and (ii) extend our study from $\mathrm{H}_{3}^{+}\left(\mathrm{H}_{2}\right)_{m=12}$ to a range of cluster sizes $(\mathrm{m}=6$ to 14$)$ thus enhancing the reliability of the overall result obtained. The experimental method involves selecting energy-specified decay reactions in high energy collisions (60 keV/amu) of hydrogen cluster ions with a helium target by utilizing event-by-event data of a recently developed multi-coincidence experiment. Assigning then corresponding temperatures to these microcanonical cluster ensembles by analyzing respective fragment size distributions, we are able to construct caloric curves for $\mathrm{H}_{3}^{+}\left(\mathrm{H}_{2}\right)_{m}$ cluster ions. Caloric curves of all projectile ions and a caloric curve of the mean of these curves show a backbending in the plateau region (which is also confirmed by a characteristic change in the shape of the corresponding fragment size distributions), thus constituting direct evidence for a negative microcanonical heat capacity in the liquid-to-gas like transition of these finite systems. In the following we will shortly describe the experimental method, then discuss the method to derive the caloric curve and finally present and discuss the results obtained for the selected cluster ions studied $\mathrm{H}_{15}^{+}$, $\mathrm{H}_{19}^{+}, \mathrm{H}_{21}^{+}, \mathrm{H}_{25}^{+}, \mathrm{H}_{27}^{+}$and $\mathrm{H}_{31}^{+}$.

As described previously [17] mass selected hydrogen cluster ions $\mathrm{H}_{3}^{+}\left(\mathrm{H}_{2}\right)_{m}$ with a velocity of $\mathrm{c} / 88$ (using different acceleration voltages in the MV range) are prepared in a high-energy cluster ion beam facility consisting of a cryogenic cluster jet expansion source combined with a high performance electron ionizer and a two step ion accelerator (consisting of an electrostatic field and a RFQ post-accelerator). After momentum analysis by a magnetic sector field, the mass selected high energy projectile pulse (pulse length:100 ms, repetition frequency:1 $\mathrm{Hz}$ ) is crossed perpendicularly by a helium target beam effusing from a cylindrical capillary tube. Prior to this, the ion beam is collimated by two apertures ensuring an angular dispersion of about $\pm 0.8 \mathrm{mrad}$. One meter behind this collision region the high energy hydrogen collision products (neutral and ionized) are passing a magnetic sector field analyzer. The undissociated primary $\mathrm{H}_{3}^{+}\left(\mathrm{H}_{2}\right)_{m \leq 14}$ cluster projectile ion or the neutral and charged fragments resulting from reactive collisions are then detected approximately $0.3 \mu$ s after the collision event with a multi-detector device consisting of an array of passivated implanted planar silicon surface barrier detectors located at different positions at the exit of the magnetic analyzer. With this instrument we are able to analyze on an event-by-event basis the identity of all correlated fragments produced in a single collision event between the $\mathrm{H}_{3}^{+}\left(\mathrm{H}_{2}\right)_{m \leq 14}$ cluster ion and the $\mathrm{He}$ target atom (for details see references given in [17] and details given in a forthcoming paper on the experimental technique [21]).

For the construction of a caloric curve we need the simultaneous determination of the energy and the temperature of the microcanonical system. The energy is determined by selecting from the many ten thousands of event subgroups representing collisions in which a certain amount of energy is deposited into the cluster. For this we need to analyze each decay reaction in terms of the energy required for all the particles produced (e.g., $\mathrm{H}_{2}^{+}$, $\mathrm{H}, \mathrm{H}^{+}$, etc.) in such a decay reaction and to use the total energy value obtained as a measure for the internal energy prior to the decay.

To plot a caloric curve we need to determine for each subgroup of known energy the corresponding temperature of the cluster ions prior to the decay. As discussed previously [17] we use for this a relationship between the characteristic shape of a fragment mass distribution and the temperature of the decaying nuclei at around the critical point reported recently by Belkacem et al. [22] using the Fisher's droplet model [23]. Belkacem et al. were able to fit numerical mass distributions generated by classical molecular dynamics (CMD) calculations for an $A=100$ nucleus at different initial canonical temperatures very well with the Fisher's droplet formula

$$
d N / d A=Y_{0} \mathrm{~A}^{-\tau} \mathrm{X}^{B} \mathrm{Y}^{A}
$$

with $\mathrm{Y}_{0}, \mathrm{X}, \mathrm{Y}$ and $\tau$ fitting parameters and $\mathrm{A}$ the atomic mass number and $B=A^{2 / 3}$, thus demonstrating a direct relationship between the shape of the mass distribution as described by (1) and the initial temperature of the decaying system. Utilizing this very methodology we also obtain very good fits for the fragment size distribution of each subgroup. This then allows us to designate for each of the subgroup considered by comparison with the results of Belkacem et al. [22] a measure for the temperature due to the characteristic shape of its fragment mass distribution, i.e., the shape of the fragment distribution for a specific projectile ion changes significantly as a function of the energy deposited, i.e., from a U-shaped form at low energy which is due to the occurrence of a mixture of evaporation and multi-fragmentation processes, to a more power like law at intermediate energies (see also a detailed discussion and examples given at the end of this paper) due to the presence of only multi-fragmentation reactions, to finally a regime at high energies which is dominated by complete disintegration processes showing an extremely steep decline. For details see a first application of this method to the data of decaying $\mathrm{H}_{27}^{+}$ions 
[17] and a forthcoming paper on experimental techniques $[21]$.

Using this "temperature" which is only a relative measure (due to the comparison with CMD calculations applied to the nuclear matter) we have plotted in Fig.1 the corresponding caloric curves. All of these caloric curves clearly show three parts, after an initially rising a kind of plateau is present before the curve is rising again. These curves therefore qualitatively agree with the typical prerequisites of a first order phase transition [24]. Looking now, however, at the details of the plateau region we can see that in each case there appears in this plateau a region (between an energy of 30 to $60 \mathrm{eV}$ ) with a negative slope (called backbending) which indicates directly the occurrence of a negative heat capacity. This phenomenon can be seen even clearer when plotting the geometric means for all clusters (see Fig.2) clearly showing this characteristic change in the slope. It is interesting to note that this behaviour is seen in the individual curve of every projectile cluster ion studied (see Fig.1) and even more so when plotting the geometric means of all clusters in Fig. 2 comprising five projectile ions (the smallest projectile ion $\mathrm{H}_{15}^{+}$has been omitted in this plot and also in Fig. 3 as deviations in the general trend in Fig.1 indicate the breakdown of the statistical behavior in accordance with other studies [25]).

In order to assess the reliability of this result (i.e., the occurrence of the backbending) it is important to note that an error analysis shows that the largest error in the present method occurs in the energy determination for the subgroups in the low energy range (below about 30 $\mathrm{eV}$ ). This is due to the fact that in this energy range collisions producing one ionized molecule and one ionized molecule plus one excited molecule cannot be distinguished from each other and thus lead to errors in the energy assignment of up to appr. $10 \mathrm{eV}$. This in turn will lead to errors in the temperature determination. Nevertheless, this uncertainty will not change the general trend of the caloric curves, i.e., an initial rise up to about $30 \mathrm{eV}$ followed by a backbending and a steep rise above $70 \mathrm{eV}$. In addition the error bars are much smaller for the data points above an energy of about $30 \mathrm{eV}$ due to the much smaller probability of ionizing/exciting three molecular units in the hydrogen cluster ion (details will discussed in a forthcoming paper [21]), see the very good agreement of data points for the different ions in the region between 30 and $70 \mathrm{eV}$.

Even more convincing evidence for the occurrence of backbending can be found by examining directly the original data on the fragment size distributions (see in Fig.3 three characteristic examples of the fragment size distributions for all five cluster ions considered here). Here the occurrence of this backbending can be clearly seen (without relying on a detailed data analysis based on a model such as Fisher's droplet model used by Belkacem et al.[22]) by the characteristic change in the fragment size distribution, i.e., for data in the energy interval 55$65 \mathrm{eV}$ we have a lower temperature because there are more larger fragments present than in the energy interval $40-50 \mathrm{eV}$ and the energy interval $70-80 \mathrm{eV}$. Thus the fragment size distribution itself can be viewed as a direct measure of the temperature of the decaying clusters, thus the change in the distributions shown in Fig. 3 constitutes a direct proof of the backbending without going through the formal assigment of temperatures using the comparison with the CMD calculations of Belkacem et al. [22].

In conclusion, the present results clearly give direct experimental evidence for the occurrence of backbending in caloric curves measured for decaying hydrogen cluster ions. This is in direct accordance with predictions based on theoretical calculations of Chomaz et al. [20] for caloric curves for the microcanonical liquid-gas phase transition using a lattice gas model (see also earlier calculations for solid-liquid transitions [6,7] also predicting backbending in caloric curves). According to [20] the occurrence of a negative heat capacity is a well-defined signal of a first order phase transition when events are sorted in constant excitation energy bins. It is interesting to note that the present observation of backbending in a caloric curve has been observed for clusters as small as $\mathrm{H}_{19}^{+}$(the only earlier indirect experimental observation of backbending pertaining to a cluster consisting of $147 \mathrm{Na}$ atoms [16]) indicating that for these small sizes statistical considerations are valid.

Work supported by the FWF, Wien, Austria, the Amadee program of the French and Austrian governments and the EU Commission, Brussels.
[1] W. Thirring, Z. Phys., 235 (1970) 339

[2] D. Lynden-Bell, Physica, A263 (1999) 293

[3] A.S. Edington, Mon. Not. R. Astr. Soc., 76 (1916) 525

[4] D. Lynden-Bell and R. Wood, Mon. Not.R. Astr. Soc., 138 (1968) 495

[5] D.H.E. Gross and H. Massmann, Nucl. Phys. A471 (1987) $393 \mathrm{c}$

[6] D.H.E. Gross, Y. Zheng and H. Massmann, Phys. Lett B 200 (1988) 397

[7] M. Bixon and J. Jortner, J. Chem. Phys., 91 (1989) 1631

[8] P. Labastie and R.L. Whetten, Phys. Rev. Lett., 65
(1990) 1567

[9] D.H.E. Gross, Rep. Prog. Phys., 53 (1990) 605

[10] J. Pochodzolla et al., Phys. Rev. Lett., 75 (1995)1040

[11] P. Chomaz and F. Gulminelli, Nucl. Phys., A647 (1999) 153

[12] Y.G. Ma et al., Phys. Lett., B390 (1997) 41

[13] M. D'Agostino et al., Nucl. Phys., A650 (1999) 329

[14] M. Schmidt et al., Phys. Rev. Lett., 79 (1997) 99

[15] T. Bachels et al., Phys. Rev. Lett., 85 (2000) 1250

[16] K. Kofman et al., Phys. Rev. Lett., 86 (2001) 1388

[17] M. Schmidt et al., Phys. Rev. Lett., 86 (2001) 1191 


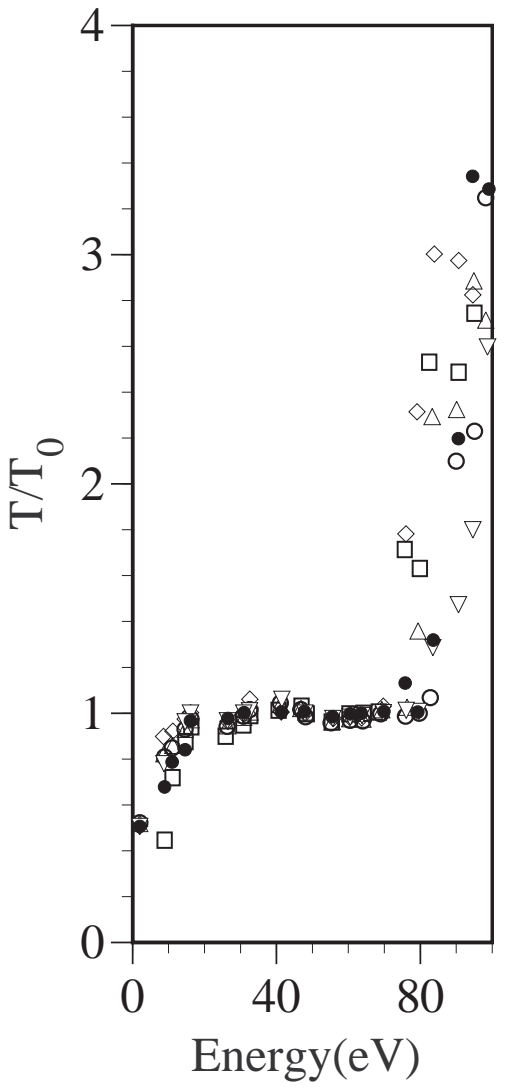

FIG. 1: Caloric curves for hydrogen cluster ion fragmentation: temperature (given in reduced values, with To the temperature in the plateau part of the curve) versus the energy deposited in the $\mathrm{H}_{3}^{+}\left(\mathrm{H}_{2}\right)_{m}$ cluster ions $(\mathrm{m}=6$ designated by open squares, $\mathrm{m}=8$ designated by open circles, $\mathrm{m}=9$ designated by open triangles, $\mathrm{m}=11$ designated by open rhombs, $\mathrm{m}=12$ designated by open inverted triangles and $\mathrm{m}=14$ designated by filled circles).
[18] M. Schmidt et al., Phys. Rev. Lett., 87 (2001) 203402

[19] F. Gobet et al., Phys. Rev. Lett., 87 (2001) 203401

[20] A.H. Raduta and A.R. Raduta, Nucl. Phys., A681 (2001) $394 \mathrm{c}$

[21] A. Bonasera, M. Bruno, C.O. Dorso and P.F. Mastinu, La Rivista del Nuovo Cimento, 23 (2000) 1

[22] P. Chomaz, V. Duflot and F. Gulminelli, Phys. Rev. Lett., 85 (2000) 3587

[23] F. Gobet et al., Phys.Rev.A, to be submitted 2001/2.

[24] M. Belkacem et al., Phys. Rev. C52 (1995) 271 M.E. Fisher, Rep. Prog.Phys., 30 (1967) 615

[25] D.H.E. Gross, Phys. Rep.279 (1997) 119

[26] S. Matt, R. Parajuli, A. Stamatovic, P. Scheier and T.D. Märk, J. Chem.Phys., in print (2002), see also R. Parajuli, S. Matt, A. Stamatovic, P. Scheier and T.D.Märk, Int.J. Mass Spectrom., in print (2002). 


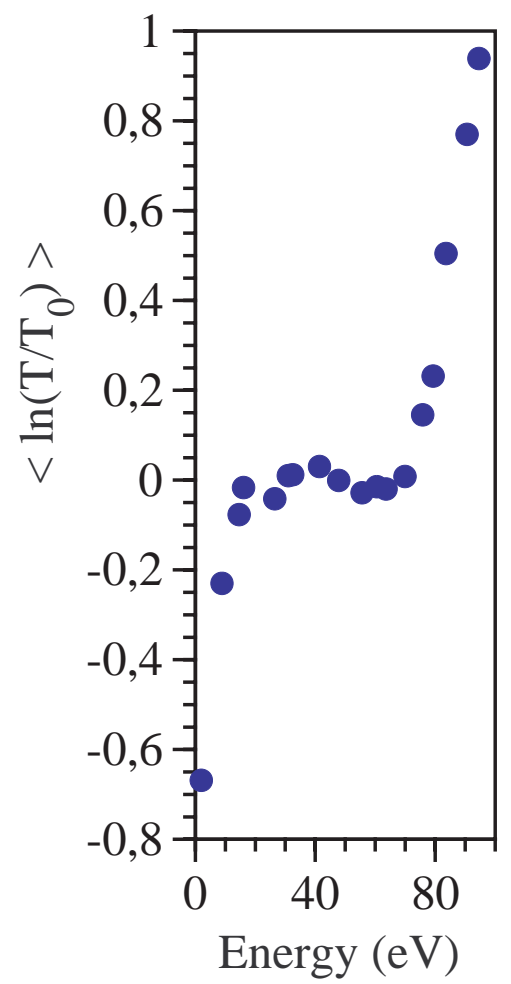

FIG. 2: Geometric means of all the curves shown in Fig.1 excluding the data for the smallest cluster ion $\mathrm{H}_{15}^{+}$(see text). 


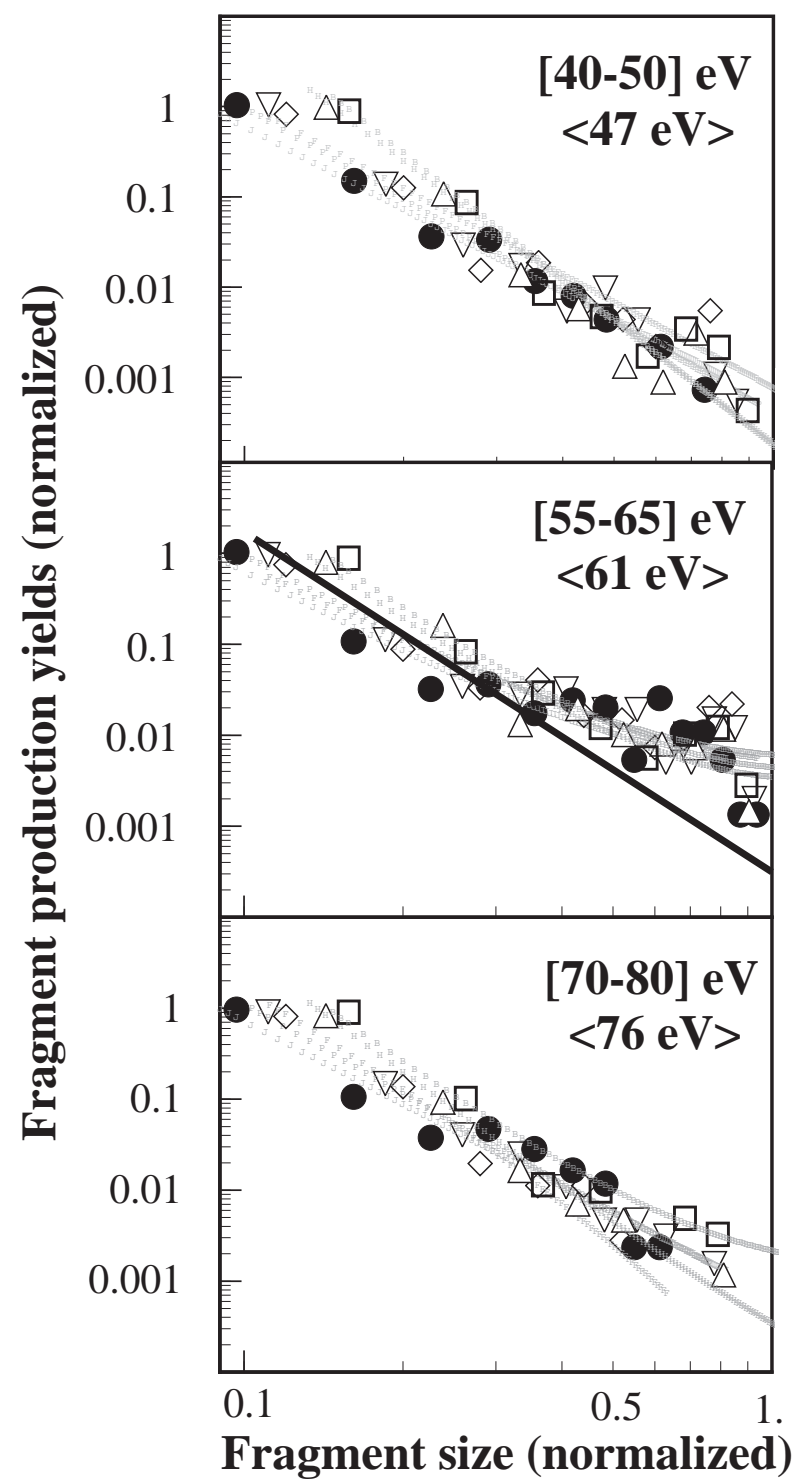

FIG. 3: Partial fragment mass distributions for subgroups selected according to the energy deposited in the collision complex occurring in collisions of $60 \mathrm{keV} / \mathrm{amu}$ projectile $\mathrm{H}_{3}^{+}\left(\mathrm{H}_{2}\right)_{m}$ ions with He corresponding to three energy ranges 40-50 eV, 55-65 eV and 70-80 eV. Dotted lines fits to the experimental data using Fisher's formula (see text), designation of symbols as in Fig.1. The line shown in the middle panel constitutes a fit to all of the data in the upper and lower panel clearly demonstrating the presence of more larger fragments in this case and therefore indicating a lower temperature for this energy range than in the upper and lower panel. 